\title{
Syndrome d'hyperviscosité : mise au point pour les réanimateurs
}

\section{Hyperviscosity Syndrome: an Update for Intensivists}

\author{
S. Valade $\cdot$ V. Lemiale $\cdot$ E. Mariotte \\ Reçu le 14 mars 2018; accepté le 20 mai 2018 \\ (C) SRLF et Lavoisier SAS 2018
}

\section{Points clés}

- Un syndrome d'hyperviscosité (SH) doit être évoqué dans un contexte d'hémopathie devant des signes neurologiques, respiratoires, cardiovasculaires ou des saignements inexpliqués.

- Il n'y a pas d'examen simple permettant de confirmer le diagnostic. Celui-ci reposera au final sur un faisceau d'arguments et un avis d'experts. L'examen le plus spécifique est le fond d'œil.

- Le SH est une urgence thérapeutique. Le traitement associe le plus souvent une chimiothérapie à un traitement symptomatique visant à diminuer la viscosité sanguine (hydratation, saignée...).
- En cas de $\mathrm{SH}$, toute intervention thérapeutique susceptible d'augmenter la viscosité sanguine doit être évitée (transfusion de globules rouges, déplétion hydrosodée...).

- Dans les formes les plus sévères de $\mathrm{SH}$, il peut être nécessaire de recourir à l'hémaphérèse thérapeutique en urgence (plasmaphérèse ou cytaphérèse), même en l'absence de diagnostic de certitude (fond d'œil non indispensable en urgence dans ce contexte).

- La réponse au traitement se juge sur la disparition des signes cliniques et l'examen du fond d'œil. La réponse au traitement fait partie des critères diagnostiques.
Résumé Le syndrome d'hyperviscosité ( $\mathrm{SH}$ ) est une entité clinique composée de signes variables non spécifiques et dont le diagnostic repose sur un faisceau d'arguments contextuels, anamnestiques et clinicobiologiques. En dehors de la mesure de la viscosité plasmatique, l'examen le plus spécifique pour confirmer le diagnostic semble être le fond d'œil. L'incidence du SH est inconnue, mais il s'agit d'une complication rare d'un grand nombre de pathologies. La physiopathologie est en rapport avec une augmentation de la viscosité plasmatique ou avec une augmentation du nombre d'éléments figurés du sang et/ou une altération de la capacité de ces cellules à circuler dans le lit capillaire. Le SH relève toujours d'un traitement de la pathologie sousjacente et d'un traitement symptomatique visant à diminuer la viscosité sanguine. Dans les formes les plus sévères, il peut être nécessaire de recourir à un traitement par aphérèse

S. Valade $\cdot$ V. Lemiale $\cdot$ E. Mariotte $(\bowtie)$

Service de médecine intensive et réanimation, hôpital Saint-Louis,

Assistance publique des Hôpitaux de Paris,

1, avenue Claude-Vellefaux, F-75010 Paris, France

e-mail : eric.mariotte@aphp.fr thérapeutique en urgence. Le pronostic dépend principalement de la pathologie sous-jacente.

Mots clés Hyperviscosité · Réanimation · Aphérèse thérapeutique

\begin{abstract}
Hyperviscosity syndrome (HS) is a clinical entity. It consists of various aspecific symptoms and its diagnosis relies on a set of contextual, anamnestic and clinicobiological arguments. Besides the measure of plasmatic viscosity, the most specific diagnostic tool seems to be the fundus examination. The incidence of HS is unknown as it is a rare complication of a wide range of diseases. The pathophysiology of the syndrome may be either related to an increase in plasma viscosity or to an elevation of the number of blood cells and/or an alteration of their capacity to pass through the capillaries. HS always requires a symptomatic treatment in order to decrease blood viscosity. In severe cases, urgent therapeutical apheresis may be necessary. The prognosis of HS depends on the underlying etiology.
\end{abstract}

Keywords Hyperviscosity · Therapeutical apheresis · Intensive care 


\section{Introduction}

Le syndrome d'hyperviscosité ( $\mathrm{SH}$ ) est une complication rare, mais potentiellement grave, de nombreuses pathologies, principalement hématologiques [1,2]. Dans cette mise au point, nous allons aborder les éléments permettant de l'évoquer, de le confirmer et de le traiter en urgence, dans les services de médecine intensive et réanimation pour les formes les plus sévères.

\section{Viscosité : une notion physique}

La viscosité d'un fluide correspond à la résistance qu'oppose ce fluide à son écoulement. Elle est donc l'inverse de sa fluidité. La viscosité d'un fluide se mesure à l'aide de sa vitesse d'écoulement dans un viscosimètre à température donnée [3]. Elle est rapportée à celle de l'eau à même température et exprimée en poises.

Le plasma sanguin est un fluide newtonien pour lequel la mesure de la viscosité est fiable et reproductible (car indépendante des conditions d'écoulement). À l'inverse, le sang est un fluide non newtonien, assimilable à une suspension cellulaire dans du plasma, pour lequel on ne dispose pas de technique universellement validée pour la mesure de la viscosité $[4,5]$.

La viscosité normale du plasma est de l'ordre de 1,41,8 centipoise [6].

\section{Syndrome d'hyperviscosité : une entité clinique}

Le SH désigne l'ensemble des manifestations en rapport avec l'augmentation de la viscosité du sang [1].

On distingue schématiquement les $\mathrm{SH}$ plasmatiques (SHP), en rapport avec l'élévation du taux de protéines dans le plasma, et les SH cellulaires (SHC), en rapport avec une augmentation du nombre d'éléments figurés dans le sang et/ ou une altération de leur capacité à circuler dans le lit capillaire (Tableau 1) [4].

Le SH, quelle que soit son étiologie, est une urgence diagnostique et thérapeutique en soins intensifs hématologiques.

\section{Physiopathologie du syndrome d'hyperviscosité plasmatique}

Le SHP est généralement en rapport avec la présence d'une immunoglobuline monoclonale en excès. Il s'agit d'une complication rare des gammapathies monoclonales dont la prévalence n'est pas précisément connue. Le SHP s'observe plus fréquemment au cours de la maladie de Waldenström (10-30\%) qu'au cours du myélome multiple (2-6\%) pour des raisons de propriétés physicochimiques de la paraprotéine produite [7]. En effet, les IgM monoclonales, de par leur structure pentamérique, présentent un poids moléculaire beaucoup plus important que celui des autres immunoglobulines (IgM : $925 \mathrm{kDa}, \mathrm{IgG}: 125 \mathrm{kDa}$, albumine : $65 \mathrm{kDa}$ ). L'augmentation du taux plasmatique d'IgM fait augmenter la viscosité de manière exponentielle, alors que la hausse du taux d'IgG la fait augmenter de manière linéaire [1]. Cela explique que les IgM monoclonales soient responsables d'une augmentation plus importante de la viscosité par rapport aux IgG pour un même taux circulant, et que des variations faibles du taux d'IgM circulant puissent entraîner des variations importantes de viscosité. Il est à noter qu'au cours des autres lymphoproliférations à différenciation plasmocytaire (lymphomes B, habituellement de bas grade), on peut également observer une production d'immunoglobuline monoclonale en quantité généralement faible, insuffisante pour entraîner un $\mathrm{SH}$.

On considère que le SHP survient pour des viscosités plasmatiques supérieures à 4 centipoises, avec des variations interindividuelles importantes [8].

Il a été décrit des SHP au cours des cryoglobulinémies, du fait de la faculté de ces immunoglobulines à se polymériser au froid. Les symptômes de SHP sont toutefois à différencier des manifestations des cryoglobulinémies en rapport avec la précipitation des complexes d'immunoglobulines avec activation du complément au niveau de la paroi des vaisseaux (= vascularite nécrosante des petits vaisseaux) [9].

Des cas de SH ont été exceptionnellement rapportés au cours des hypergammaglobulinémies polyclonales importantes associées à diverses pathologies (syndrome de Gougerot-Sjögren [10], maladie de Castleman multicentrique, etc.) ou conditions (traitements par immunoglobulines polyvalentes intraveineuses [11]).

\section{Physiopathologie du syndrome d'hyperviscosité cellulaire}

Au cours de certaines hémopathies (leucémies aiguës myéloblastiques [LAM] hyperleucocytaires, leucémie myéloïde chronique, polyglobulie de Vaquez, etc.), on peut observer une augmentation pathologique du nombre de cellules sanguines circulantes. Cela entraîne toutefois rarement un $\mathrm{SH}$ dit « cellulaire » (SHC). En effet, l'apparition du SHC nécessite une augmentation importante d'un taux de cellules de grande taille ou présentant des anomalies membranaires altérant leur capacité de circulation dans les capillaires sanguins $[12,13]$. Aussi, s'il est par exemple habituel d'observer une augmentation de la viscosité sanguine chez les patients atteints de polyglobulie de Vaquez, la présence d'un SHC est exceptionnelle dans cette population [14]. Il est également très rare de l'observer au cours de la leucémie lymphoïde chronique, où les cellules tumorales sont des lymphocytes matures, de petite taille, sans anomalies de membrane et donc capables de circuler aisément dans le lit 


\begin{tabular}{|c|c|c|c|}
\hline $\begin{array}{l}\text { Type de syndrome } \\
\text { d'hyperviscosité }\end{array}$ & Pathologie & Protéine/cellule responsable & Examens complémentaires \\
\hline \multirow[t]{4}{*}{ Plasmatique } & $\begin{array}{l}\text { Maladie de Waldenström } \\
\text { (fréquent) }\end{array}$ & IgM monoclonale > $30 \mathrm{~g} / 1$ & $\begin{array}{l}\text { Protidémie }(\uparrow) \\
\text { Électrophorèse des protides }\end{array}$ \\
\hline & Myélome multiple (rare) & $\begin{array}{l}\text { IgG monoclonale }>40 \mathrm{~g} / \mathrm{l}, \\
\mathrm{A}>60 \mathrm{~g} / \mathrm{l}, \mathrm{D}, \mathrm{E}\end{array}$ & $\begin{array}{l}\text { (pic monoclonal) } \\
\text { Immunoélectrophorèse des protides } \\
\text { (pic } \operatorname{IgM}, \operatorname{IgA}, \operatorname{IgD}, \operatorname{IgE} \text { ) }\end{array}$ \\
\hline & $\begin{array}{l}\text { Cryoglobulinémie, polyarthrite } \\
\text { rhumatoïde (très rare) }\end{array}$ & $\begin{array}{l}\text { Complexes immuns (Ig } \\
\text { monoclonale-monoclonale, } \\
\text { monoclonale-polyclonale, } \\
\text { polyclonale-polyclonale) }\end{array}$ & $\begin{array}{l}\text { Protidémie }(\uparrow) \\
\text { Recherche cryoglobuline (tube acheminé } \\
\left.\text { au laboratoire à } 37^{\circ} \mathrm{C}\right) \\
\text { Facteur rhumatoïde }\end{array}$ \\
\hline & $\begin{array}{l}\text { Syndrome de Gougerot- } \\
\text { Sjögren, maladie de Castleman, } \\
\text { VIH (très rare) }\end{array}$ & IgG polyclonales & $\begin{array}{l}\text { Électrophorèse des protides } \\
\text { (hypergammaglobulinémie polyclonale) } \\
\text { Autoanticorps } \\
\text { Sérologie/PCR HHV8 } \\
\text { Sérologie VIH }\end{array}$ \\
\hline \multirow[t]{5}{*}{$\begin{array}{l}\text { Cellulaire } \\
\text { quantitatif }\end{array}$} & $\begin{array}{l}\text { LAM hyperleucocytaire } \\
\text { (fréquent) }\end{array}$ & $\begin{array}{l}\text { Blastes myéloïdes > } 100 \mathrm{G} / 1 \\
\text { (contingent monoblastique M4 } \\
\text { et M5) }\end{array}$ & $\begin{array}{l}\text { NFS + frottis sanguin } \\
\text { Immunophénotypage (myélogramme, } \\
\text { cytoponction ganglionnaire...) }\end{array}$ \\
\hline & LAL hyperleucocytaire (rare) & Blaste lymphoïde > 300 G/1 & \\
\hline & LMC (rare) & Myélémie équilibrée $>300$ G/1 & \\
\hline & LLC (très rare) & Lymphocytes matures $>500 \mathrm{G} / 1$ & \\
\hline & $\begin{array}{l}\text { Polyglobulie de Vaquez, } \\
\text { polyglobulies secondaires (très } \\
\text { rares) }\end{array}$ & Globules rouges & NFS \\
\hline \multirow[t]{2}{*}{ Cellulaire qualitatif } & Drépanocytose (très rare) & Globules rouges falciformés & $\begin{array}{l}\text { NFS + frottis sanguin } \\
\text { Électrophorèse de l'hémoglobine } \\
\text { Dosage d'HbS }\end{array}$ \\
\hline & $\begin{array}{l}\text { Sphérocytose héréditaire, } \\
\text { thalassémie majeure (très rare) }\end{array}$ & Globules rouges rigides & $\begin{array}{l}\text { Électrophorèse de l'hémoglobine } \\
\text { Études membranes globules rouges }\end{array}$ \\
\hline
\end{tabular}

capillaire même à des taux très élevés. Pour la même raison, on n'observe jamais de SHC au cours des hyperleucocytoses à polynucléaires neutrophiles fréquemment présentes au cours des syndromes infectieux graves.

Le SH compliquant les leucémies aiguës est appelé leucostase. En plus des manifestations habituelles de $\mathrm{SH}$, on peut observer des anomalies en rapport avec la capacité des blastes à adhérer à l'endothélium capillaire, essentiellement pulmonaire, indépendamment du taux de leucocytes [15].

Enfin, chez les patients avec des hémoglobinopathies telles que la drépanocytose, on peut exceptionnellement observer durant les crises, en dehors des manifestations liées à l'hémolyse au premier plan, des SHC « qualitatifs » causés par les globules rouges falciformés ayant perdu leur élasticité [16].

\section{Diagnostic}

\section{Anamnèse et clinique}

Le SH se manifeste par un cortège de symptômes non spécifiques qu'il convient de rechercher à l'interrogatoire et à l'examen clinique.

La triade de Waldenström, décrite initialement en 1944, associe des signes visuels, des hémorragies et des signes neurologiques [1]. La sensibilité de la triade de Waldenström pour le diagnostic de SH était dans cette étude de l'ordre de $70 \%$.

Les signes ophtalmologiques peuvent consister en une baisse de l'acuité visuelle, des phosphènes et des myodésopsies. Le fond d'œil doit être systématiquement réalisé devant une suspicion de SH. Il peut mettre en évidence des 
signes évocateurs avec une dilatation des vaisseaux rétiniens " en chapelet de saucisses », des hémorragies rétiniennes, des nodules cotonneux et des microanévrismes. Dans les formes les plus sévères, il peut y avoir une thrombose de la veine centrale de la rétine et un œdème papillaire. La sévérité de l'atteinte rétinienne a été corrélée à la viscosité plasmatique [17]. Le fond d'œil étant la plupart du temps difficile à obtenir en urgence, on peut être amené à débuter le traitement d'une suspicion de SH sévère sans attendre la réalisation de cet examen.

Du fait de la grande sensibilité de cet examen, on peut considérer qu'un fond d'œil normal permet d'exclure le diagnostic de SH. Toutefois, il s'agit d'un examen parfois compliqué (coopération du patient, ininterprétable en cas de cataracte, etc.) et opérateur-dépendant, ce qui doit conduire à son contrôle en cas de forte suspicion de SH.

Le syndrome hémorragique est habituellement surtout cutanéomuqueux avec des épistaxis et des ecchymoses au premier plan. Il est en rapport le plus souvent avec des anomalies des fonctions plaquettaires du fait de l'hyperprotidémie et peut être majoré par une éventuelle coagulopathie associée.

Parmi les troubles neurologiques en rapport avec les $\mathrm{SH}$, on note un ralentissement psychomoteur, des céphalées, des acouphènes, des vertiges avec ou sans ataxie. Dans les cas les plus sévères, on peut observer des syndromes confusionnels, des comas et des accidents vasculaires cérébraux $[1,18]$.

On peut également observer des signes cardiovasculaires et respiratoires. L'hyperviscosité va en effet favoriser la stase et les thromboses. L'hémodilution peut se compliquer d'un tableau d'insuffisance cardiaque avec œdème aigu pulmonaire de surcharge [19].

Le syndrome de leucostase est le SHC associé aux hémopathies agressives, essentiellement leucémies aiguës hyperleucocytaires. On peut y observer plus volontiers que dans les autres types de $\mathrm{SH}$ des signes respiratoires sévères, des hémorragies intracérébrales et un priapisme. Il est souvent difficile de savoir quelle part des signes cliniques revient au SH et quelle part revient aux troubles de la coagulation induits par l'hémopathie (thrombopénie centrale, CIVD, etc.), à l'éventuel syndrome de lyse tumorale et à l'infiltration des organes par les cellules cancéreuses [15].

On observe souvent des signes généraux à type d'asthénie. Peuvent s'y associer les signes de l'éventuelle hémopathie sous-jacente (fièvre, sueurs nocturnes, anorexie).

\section{Biologie standard}

En dehors de la mesure de la viscosité plasmatique, non réalisée en routine, certains éléments biologiques peuvent étayer un diagnostic de $\mathrm{SH}$.
L'impossibilité d'obtenir des résultats biologiques à partir des prélèvements sanguins peut s'observer dans les formes les plus sévères de $\mathrm{SH}$ avec des difficultés techniques de prélèvement et une hémolyse rapide dans les tubes de sang. Cela s'observe en particulier dans le cadre des hémopathies myéloïdes de haut grade (leucémies aiguës) où les prélèvements sanguins doivent être acheminés et traités rapidement aux laboratoires du fait de modifications importantes in vitro.

En cas de SHP, la présence d'une grande hyperprotidémie est évocatrice, à plus forte raison quand elle s'associe à la présence d'hématies en rouleaux sur le frottis sanguin [20].

En cas de SHC, on peut observer au frottis sanguin une hyperleucocytose composée de cellules anormales au cours des hémopathies ou des hématies falciformées au cours des crises vaso-occlusives de la drépanocytose.

Il peut y avoir, du fait de l'hyperviscosité, des stigmates biologiques de dysfonctionnement de tous les organes (altération des échanges gazeux, insuffisance rénale aiguë, CIVD, etc.).

\section{Biologie spécialisée}

Comme précédemment évoqué, l'hyperviscosité plasmatique peut être confirmée par une mesure de la viscosité [1].

Dans le cadre des SHP, l'électrophorèse des protéines sériques montre une hypergammaglobulinémie le plus souvent monoclonale. Ce pic monoclonal peut être caractérisé par immunofixation. Il est admis que l'on n'observe habituellement pas de SHP pour des taux d'IgG monoclonale inférieurs à $40 \mathrm{~g} / \mathrm{l}$, des taux d'IgA inférieurs à $60 \mathrm{~g} / \mathrm{l}$, des taux d'IgM inférieurs à $30 \mathrm{~g} / \mathrm{l}$ [21]. Il est à noter qu'il existe de grandes variabilités interindividuelles dans le seuil de survenue de SHP, certains patients " tolérant » des taux extrêmement élevés de paraprotéines. En revanche, pour un même patient, le seuil du taux de protéines pour lequel les signes cliniques réapparaissent est assez reproductible. La présence d'une activité cryoglobuline chez les immunoglobulines en excès peut rendre les patients symptomatiques à des seuils plus bas que ceux précédemment indiqués [22]. La recherche de cryoglobuline est à effectuer sur des tubes rapidement acheminés au laboratoire à $37^{\circ} \mathrm{C}$.

Dans le cadre des SHC, la formule sanguine et le frottis sanguin permettent d'identifier les cellules anormales en excès (blastes, globules rouges, lymphocytes matures, etc.). Le bilan peut être complété par un myélogramme, une analyse phénotypique des cellules circulantes, une recherche de clonalité, un caryotype et une analyse en biologie moléculaire. Il est plus fréquent d'observer des SHC (leucostase) au cours des LAM par rapport aux LAL et au sein des LAM au cours de celles s'accompagnant d'un contingent monoblastique (LAM 4 et 5). 


\section{Traitement}

\section{Traitement symptomatique}

Le traitement symptomatique du $\mathrm{SH}$ vise à diminuer rapidement la viscosité du sang soit en diminuant le taux de protides, soit en diminuant le nombre d'éléments figurés.

Il repose dans un premier temps sur l'hyperhydratation intraveineuse quand cela est possible.

Il convient également de limiter au maximum toute intervention thérapeutique susceptible d'augmenter la viscosité sanguine. On pense en particulier à la transfusion de globules rouges et à la déplétion par diurétiques devant des tableaux d'insuffisance respiratoire aiguë ou d'insuffisance cardiaque congestive en rapport avec l'hyperviscosité [23]. Une anémie même profonde doit donc être tolérée en l'absence de signe de mauvaise tolérance.

Pour les SHC liés à une polyglobulie ou à une pathologie du globule rouge, la réalisation de saignées permet la diminution rapide de l'hématocrite et donc de la viscosité sanguine. Un échange transfusionnel par saignée-transfusion de globules rouges peut être indiqué chez les patients drépanocytaires [24].

En cas de $\mathrm{SH}$ sévère, un recours à l'aphérèse thérapeutique est requis en urgence.

Un résumé des traitements des $\mathrm{SH}$ et des modalités de surveillance de leur efficacité est proposé dans le tableau 2.

\section{Plasmaphérèse au cours des SHP}

La plasmaphérèse est une technique d'épuration extrarénale où l'on prélève le plasma du patient pour le remplacer volume par volume par un fluide de substitution. Il s'agit d'un traitement symptomatique rapidement efficace pour les SHP puisqu'il permet la diminution rapide du taux de protéines pathologiques dans le plasma du patient sans risque de surcharge vasculaire $[25,26]$. Deux techniques sont disponibles et se distinguent par la méthode de séparation du plasma du patient par hémofiltration ou par centrifugation. Ces deux techniques, si elles sont considérées comme équivalentes en termes d'efficacité [27], ont des avantages et des inconvénients : possibilité de s'effectuer avec la plupart des générateurs d'épuration extrarénale continue, sur un cathéter de dialyse, sous anticoagulation locorégionale ou systémique pour la filtration ; nécessité de générateurs dédiés à l'aphérèse thérapeutique, sur deux voies veineuses périphériques ou un cathéter de dialyse, sous anticoagulation locorégionale pour la centrifugation.

Les échanges plasmatiques doivent être débutés en urgence dans les formes sévères de $\mathrm{SH}$, souvent sans attendre la confirmation du diagnostic de SH par le fond d'œil.

On réalise dans cette indication un à trois échanges plasmatiques espacés de 24 à 48 heures. Le volume d'échange est de 1 à 1,5 masse sanguine (40-60 ml/kg). Le fluide de substitution est généralement un colloïde de type albumine $5 \%$. L'efficacité du traitement est évaluée sur la clinique avec la disparition des signes d'hyperviscosité, notamment au fond d'œil qui doit être répété, si cela est possible [20,28]. Il est également habituel de contrôler le taux de protides et/ ou l'électrophorèse des protides après échange pour vérifier l'efficacité et pour connaître le seuil de tolérance clinique du patient.

Quelques limites sont à connaître. L'efficacité de l'élimination des immunoglobulines dépend de leur poids moléculaire, de leur volume de distribution et de leur répartition intravasculaire. Ainsi, il est beaucoup plus facile de faire baisser significativement le taux d'une $\operatorname{IgM}$ monoclonale (haut poids moléculaire, répartition intravasculaire : $90 \%$ ) que celui d'une IgG (poids moléculaire plus faible, répartition intravasculaire : $40 \%$ ). Il faut donc généralement plus

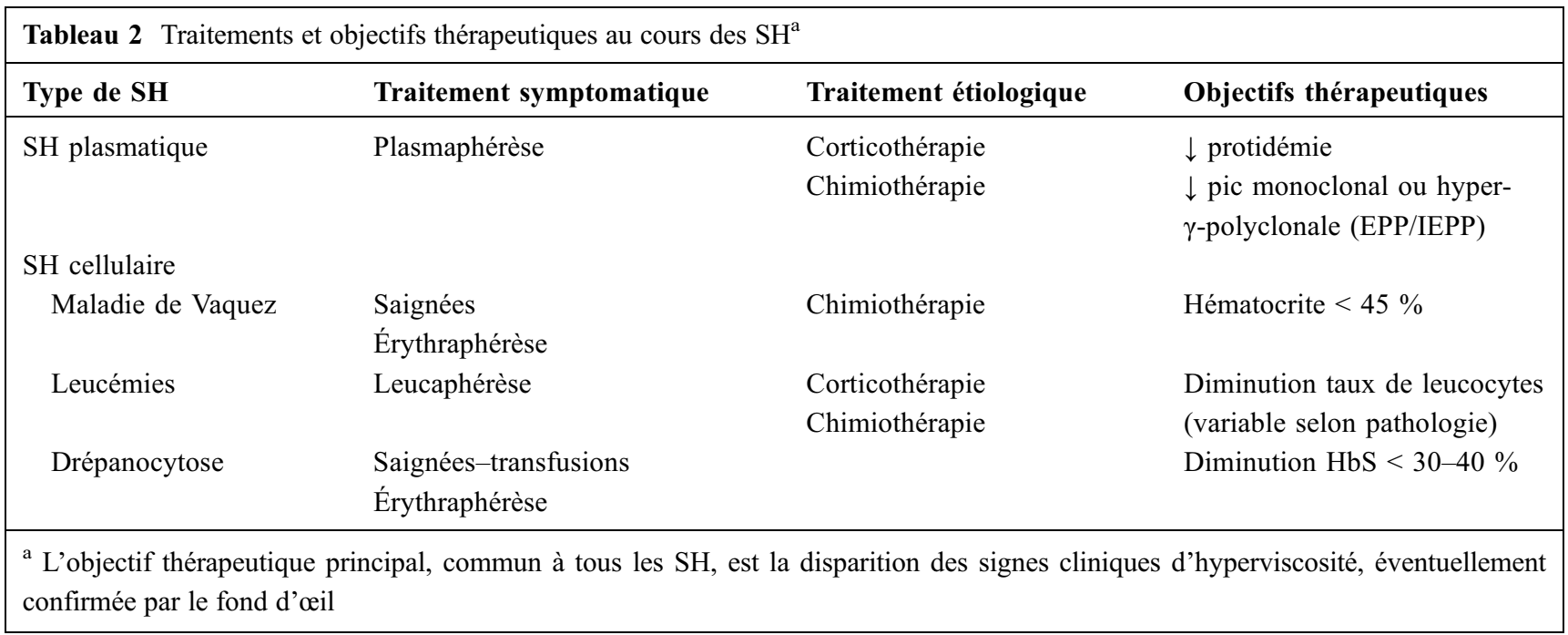


d'échanges plasmatiques pour corriger un SHP au cours d'un myélome multiple qu'au cours d'une maladie de Waldenström [29].

En cas de syndrome hémorragique important en rapport avec le $\mathrm{SH}$, il est habituel de réaliser tout ou partie de l'échange plasmatique en utilisant du plasma issu de donneur comme fluide de substitution. En cas d'échange « mixte » colloïde-plasma, on administre le plasma en dernier afin d'échanger le moins possible les facteurs de coagulation apportés [28].

\section{Cytaphérèse au cours des SHC}

Certaines sociétés savantes recommandent toujours la réalisation de cytaphérèses pour la prise en charge des syndromes de leucostase associés aux hémopathies malignes (leucaphérèse) [30]. Toutefois, la difficulté de mise en œuvre de ces techniques et l'effet controversé sur le pronostic des patients font qu'en France la cytoréduction par hydroxyurée éventuellement associée aux corticostéroïdes reste la méthode la plus utilisée pour la prise en charge du SH associé aux hémopathies agressives (LAM).

Chez les patients drépanocytaires présentant des comorbidités cardiovasculaires, neurologiques ou rénales sévères, on peut avoir recours à l'érythraphérèse qui consiste à prélever les hématies du patient remplacées par des globules rouges de donneurs sains à volume constant (contrairement aux saignées-transfusions) [24].

La plasmaphérèse n'a bien entendu pas sa place dans le traitement des SHC.

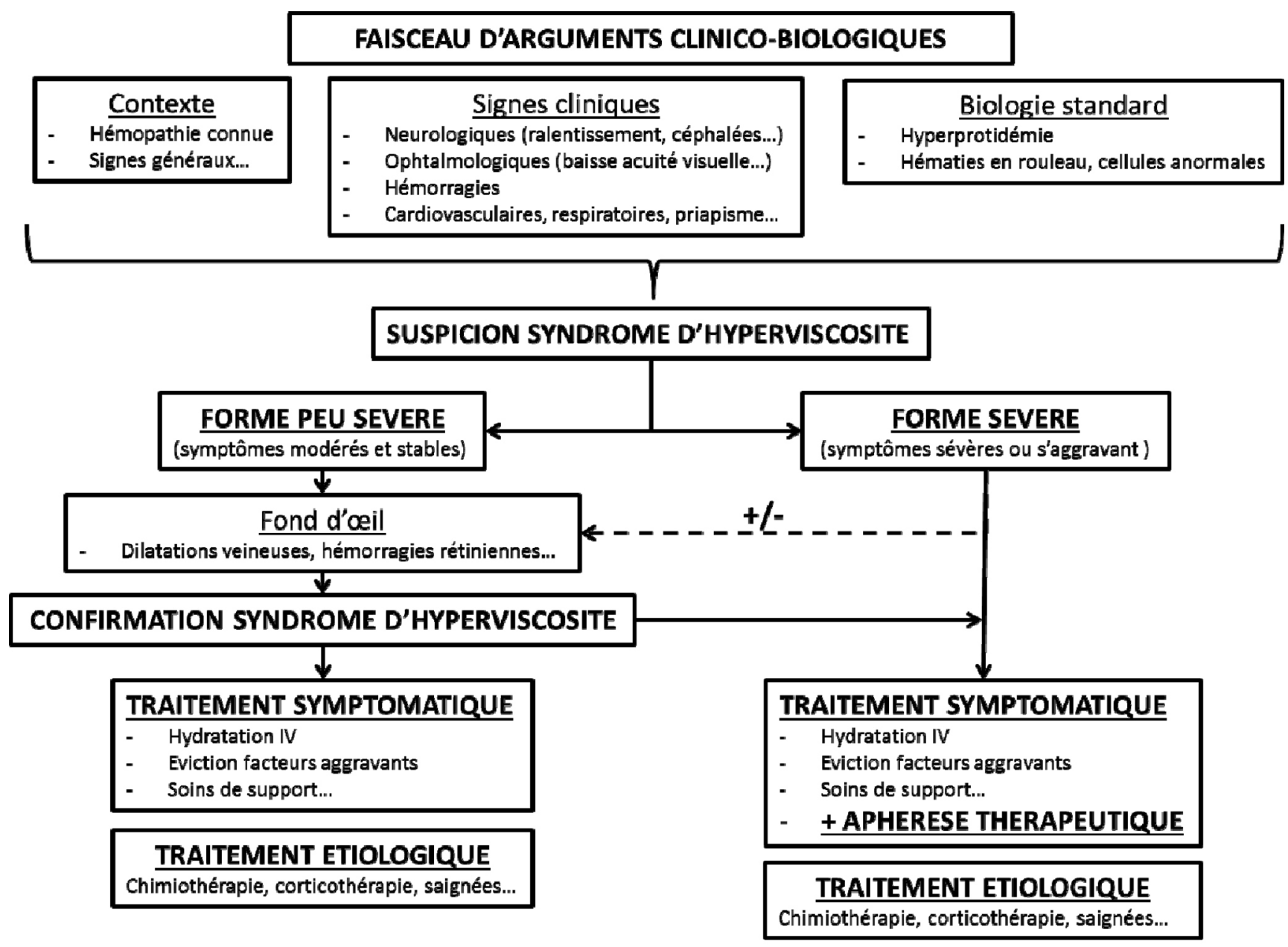

(En cas de suspicion de forme sévère de $\mathrm{SH}$, on peut être amené à instaurer un traitement symptomatique en l'absence de fond d'œil, si celui-ci n'est pas rapidement disponible.)

Fig. 1 Algorithme de prise en charge d'une suspicion de syndrome d'hyperviscosité 


\section{Traitement étiologique}

Le traitement des SH repose toujours sur le traitement en urgence de la pathologie sous-jacente, avec le plus souvent l'initiation d'une chimiothérapie anticancéreuse et/ou une corticothérapie à forte dose.

\section{Conclusion}

La grande diversité des mécanismes en cause dans le SH, l'absence de signe clinique pathognomonique et de méthode de mesure de la viscosité sanguine facilement accessible en routine rendent difficile l'élaboration de recommandations standardisées pour la prise en charge des SH. Un algorithme peut être proposé selon la gravité du SH suspecté (Fig. 1). Le diagnostic repose sur un faisceau d'arguments cliniques et biologiques dont la clé de voûte est l'examen du fond d'œil. La recherche d'une gammapathie monoclonale, essentiellement $\mathrm{IgM}$, est une étape incontournable du bilan étiologique. Le recours à des avis spécialisés est parfois nécessaire, mais il reste important de faire rapidement le diagnostic de SH pour éviter les facteurs aggravants et la survenue des complications graves. Ainsi, les réanimateurs doivent savoir décider seuls de l'initiation en urgence d'un traitement symptomatique par échanges plasmatiques en cas de forte suspicion de SHP sévère.

Des études en médecine intensive et réanimation sont nécessaires pour définir la prise en charge optimale des patients les plus sévères.

Liens d'intérêts : les auteurs déclarent ne pas avoir de lien d'intérêt.

\section{Références}

1. Fahey JL, Barth WF, Solomon A, (1965) Serum hyperviscosity syndrome. JAMA 192: 464-467

2. Gertz MA, Kyle RA, (1995) Hyperviscosity syndrome. J Intensive Care Med 10: 128-141

3. Zimmerman E, (1967) Automatic recording of viscosity changes with the Ostwald capillary viscometer. Anal Biochem 21: 81-85

4. Wells R, (1970) Syndromes of hyperviscosity. N Engl J Med 283: 183-186

5. Dumas G, Merceron S, Zafrani L, Canet E, Lemiale V, Kouatchet A, Azoulay E, (2015) Hyperviscosity syndrome. Rev Med Interne 36: 588-595

6. Perry MC, Hoagland HC, (1976) The hyperviscosity syndrome. JAMA 236: 392-393

7. Kwaan HC, (2013) Hyperviscosity in plasma cell dyscrasias. Clin Hemorheol Microcirc 55: 75-83

8. Merci de fournir les auteurs]] (1971) Hyperviscosity syndrome. Br Med J 2: 184-185

9. Retamozo S, Brito-Zerón P, Bosch X, Stone JH, Ramos-Casals M, (2013) Cryoglobulinemic disease. Oncology (Williston Park, NY) 27: 1098-1105 (1110-1116)

10. Hernández-Molina G, Bermúdez-Bermejo P, (2017) Hyperviscosity in primary Sjögren's syndrome: clinical implications. Int $\mathrm{J}$ Rheum Dis 20: 84-89
11. Hague RA, Eden OB, Yap PL, Mok JY, Rae P, (1990) Hyperviscosity in HIV infected children--a potential hazard during intravenous immunoglobulin therapy. Blut 61: 66-67

12. Merci de fournir les auteurs]] (2003) Hyperviscosity as a complication in a variety of disorders. Semin Thromb Hemost 29: 459-466

13. Adams BD, Baker R, Lopez JA, Spencer S, (2010) Myeloproliferative disorders and the hyperviscosity syndrome. Hematol Oncol Clin North Am 24: 585-602

14. Lo Presti R, Caracciolo C, Montana M, Barone R, Catania A, Caimi G, (2012) Erythrocyte deformability evaluated by laser diffractometry in polycythemia vera. Clin Hemorheol Microcirc 50: 189-192

15. Porcu P, Cripe LD, Ng EW, Bhatia S, Danielson CM, Orazi A, McCarthy LJ, (2000) Hyperleukocytic leukemias and leukostasis: a review of pathophysiology, clinical presentation and management. Leuk Lymphoma 39: 1-18

16. Bartolucci P, Lionnet F, (2014) Chronic complications of sickle cell disease. Rev Prat 64: 1120-1126

17. Menke MN, Feke GT, McMeel JW, Treon SP, (2009) Ophthalmologic techniques to assess the severity of hyperviscosity syndrome and the effect of plasmapheresis in patients with Waldenström's macroglobulinemia. Clin Lymphoma Myeloma 9: 100-103

18. Jo JT, Schiff D, (2017) Management of neuro-oncologic emergencies. In: Handbook of Clinical Neurology. Elsevier, pp 715-741

19. Shenoy S, Shetty S, Lankala S, Anwer F, Yeager A, Adigopula S, (2017) Cardiovascular oncologic emergencies. Cardiology 138: $147-158$

20. Stone MJ, Bogen SA, (2012) Evidence-based focused review of management of hyperviscosity syndrome. Blood 119: 2205-2208

21. Mehta J, Singhal S, (2003) Hyperviscosity syndrome in plasma cell dyscrasias. Semin Thromb Hemost 29: 467-471

22. Stone MJ, (2009) Waldenström's macroglobulinemia: hyperviscosity syndrome and cryoglobulinemia. Clin Lymphoma Myeloma 9: 97-99

23. Lewis MA, Hendrickson AW, Moynihan TJ, (2011) Oncologic emergencies: pathophysiology, presentation, diagnosis, and treatment. CA Cancer J Clin 61: 287-314

24. Valbonesi M, Bruni R, (2000) Clinical application of therapeutic erythrocytapheresis (TEA). Transfus Sci 22: 183-194

25. Kalayoglu-Besisik S, (2018) The use of emergency apheresis in the management of plasma cell disorders. Transfus Apher Sci. doi: $10.1016 /$ j.transci.2018.02.014

26. Castillo JJ, Garcia-Sanz R, Hatjiharissi E, Kyle RA, Leleu X, McMaster M, Merlini G, Minnema MC, Morra E, Owen RG, Poulain S, Stone MJ, Tam C, Varettoni M, Dimopoulos MA, Treon SP, Kastritis E, (2016) Recommendations for the diagnosis and initial evaluation of patients with Waldenström macroglobulinaemia: a Task Force from the 8th International Workshop on Waldenström macroglobulinaemia. Br J Haematol 175: 77-86

27. Gurland HJ, Lysaght MJ, Samtleben W, Schmidt B, (1984) A comparison of centrifugal and membrane-based apheresis formats. Int J Artif Organs 7: 35-38

28. Schwartz J, Padmanabhan A, Aqui N, Balogun RA, ConnellySmith L, Delaney M, Dunbar NM, Witt V, Wu Y, Shaz BH, (2016) Guidelines on the use of therapeutic apheresis in clinical practice-evidence-based approach from the writing Committee of the American Society for Apheresis: the Seventh Special Issue: therapeutic apheresis-guidelines 2016. J Clin Apher 31: 149-162

29. Kaplan AA, (2013) Therapeutic plasma exchange: a technical and operational review: therapeutic plasma exchange. J Clin Apher 28: 3-10

30. Aqui N, O'Doherty U, (2014) Leukocytapheresis for the treatment of hyperleukocytosis secondary to acute leukemia. Hematology Am Soc Hematol Educ Program 2014: 457-460 